\title{
AN ADVANCE IN INFINITE GRAPH MODELS FOR THE ANALYSIS OF TRANSPORTATION NETWORKS
}

\author{
MARTÍN CERA $^{a, *}$, EUGENIO M. FEDRIANI ${ }^{b}$ \\ ${ }^{a}$ Department of Applied Mathematics I \\ University of Seville, ETSIA, Ctra. Utrera km 1, ES-41013 Seville, Spain \\ e-mail: mcera@us.es \\ ${ }^{b}$ Department of Economics, Quantitative Methods and Economic History \\ Pablo de Olavide University, Ctra. Utrera km 1, ES-41013 Seville, Spain \\ e-mail: efedmar@upo.es
}

\begin{abstract}
This paper extends to infinite graphs the most general extremal issues, which are problems of determining the maximum number of edges of a graph not containing a given subgraph. It also relates the new results with the corresponding situations for the finite case. In particular, concepts from 'finite' graph theory, like the average degree and the extremal number, are generalized and computed for some specific cases. Finally, some applications of infinite graphs to the transportation of dangerous goods are presented; they involve the analysis of networks and percolation thresholds.
\end{abstract}

Keywords: infinite graph, average degree, extremal problems, road transport network, percolation.

\section{Introduction}

Graph theory is a very useful tool in various fields of human knowledge. However, when trying to solve real problems, scientists may need to develop the existing theory beyond the point it has reached so far. For instance, the analysis of continuously increasing networks, extremely complex systems, fluids filtering through porous materials, etc. requires the involvement of infinite graphs and some properties that have hitherto been developed only for the finite case.

Maybe the most clear example of this fact is the average degree. In general, and not only in extremal graph theory, we can find many problems involving the relationship between the numbers of vertices and edges of a graph (see, e.g., Cera et al., 2000; 2004, Yang et al., 2002; Yousefi-Azaria et al., 2011), i.e., the average degree. In some cases, many of these problems could be posed for infinite graphs.

We can find in the literature many papers studying the problem of providing a definition of the average degree for infinite graphs (see Stein, 2011; Stein and Zamora, 2013; Wierman and Naor, 2005). Up to now, no

* Corresponding author formal definition has been found. Indeed, it is not possible to give a general definition of the average degree for any infinite graph.

On the basis of the above, this paper firstly aims to relate the concepts of the infinite graph and the average degree. In fact, we define the average degree for a family of infinite graphs that we call average-measurable.

This definition allows us to extend to infinite graphs the problem of determining the maximum number of edges of a graph not containing a subgraph homeomorphic to a complete graph. We study the relationship of this problem with its counterpart in finite graphs.

Notation and terminology not explicitly given here can be found in theoretical handbooks (Diestel, 2000; Mader, 1998b; Milková, 2009).

1.1. Function $\boldsymbol{d}(\boldsymbol{p})$ for the finite case. Given $F$ as a finite graph, the extremal number $\operatorname{ex}(n ; F)$ denotes the maximum number of edges of a graph with $n$ vertices not containing $F$ as a subgraph. This definition induces the most general type of question we can state in extremal graph theory. It can be posed for finite as well as infinite graphs. The question is whether some invariant (e.g., edge-density, minimum degree, chromatic number 
or average degree) has an influence on the showing up of substructures or another graph invariant. In this context, an important, well-known result in finite graph theory implies that large average degrees in finite graphs force large minors and topological minors (a subgraph homeomorphic to a complete graph) (see Diestel, 2000). The extension of these problems from finite graphs to infinite graphs is interesting in graph theory. Some of them can even be found for some extremal problems (see Stein, 2011; Stein and Zamora, 2013).

Given a graph $G$, let us denote by $v(G)=|V(G)|$ and $e(G)=|E(G)|$ the cardinals of the vertices and edges of $G$, respectively. Then the average degree of $G$ is

$$
d(G)=\frac{2 e(G)}{v(G)} .
$$

Now, we note that the function

$$
d(p)=\inf \left\{t: d(G) \geq t \longrightarrow T K_{p} \subseteq G\right\},
$$

stated by Mader (1967), may be formulated in terms of the function ex $\left(n ; T K_{p}\right)$, i.e., in terms of the number of edges of a graph with $n$ vertices and not containing a subgraph homeomorphic to a complete graph (topological clique).

Proposition 1. Let p be a non-negative integer. Then

$$
d(p)=\sup _{n \geq p}\left\{\frac{2 \operatorname{ex}\left(n ; T K_{p}\right)}{n}\right\} .
$$

Proof. Write

$$
d\left(n ; T K_{p}\right)=\frac{2 \operatorname{ex}\left(n ; T K_{p}\right)}{n} .
$$

If

$$
d(p)>\sup _{n \geq p}\left\{d\left(n ; T K_{p}\right)\right\},
$$

then there exits a real number $t$ such that

$$
d(p)>t>\sup _{n \geq p}\left\{d\left(n ; T K_{p}\right)\right\} .
$$

Let us consider a graph $G$ satisfying $d(G) \geq t>$ $d\left(|V(G)| ; T K_{p}\right)$. Hence, $T K_{p} \subseteq G$ and, therefore, by the definition of $d(p), d(p) \leq t$, but this is not possible. Thus,

$$
d(p) \leq \sup _{n \geq p}\left\{d\left(n ; T K_{p}\right)\right\} .
$$

To prove the converse inequality, we suppose that there exists a positive integer $n$ such that $d(p)<d\left(n ; T K_{p}\right)$. Now, we consider $t_{n} \in \mathbb{R}$ such that $d(p)<t_{n}<d\left(n ; T K_{p}\right)$. Therefore, for every graph $G$ with $|V(G)|=n$, if $d(G) \geq t_{n}>d(p)$, then (by the definition of $d(p)) G$ contains a subgraph homeomorphic to $K_{p}$. Hence, by the definition of $d\left(n ; T K_{p}\right)$, we would have $d\left(n ; T K_{p}\right) \leq t_{n}<d\left(n ; T K_{p}\right)$, but this is not possible, either. Thus, $d(p) \geq d\left(n ; T K_{p}\right)$ for all $n$ and the result follows.
1.2. Paper objectives and structure. Bearing in mind the aforementioned result, if we want to get exact values for the function $d(p)$, it is sufficient to calculate exact values for $\operatorname{ex}\left(n ; T K_{p}\right)$ with $n$ being sufficiently large. In other words, since there exists a necessity of studying $\operatorname{ex}\left(n ; T K_{p}\right)$ for $n$ tending to infinite, we state the problem of studying this function for infinite graphs.

Additionally, the problem of studying the number of edges in relation to the number of vertices in an infinite graph has no sense. But this paper explains that it is useful as a local concept when dealing with transport networks. This fact suggests the possibility of considering the idea of defining the concept of an average degree for infinite graphs. This idea seems even more interesting if we bear in mind that an infinite graph may be considered the limit of finite graphs.

Taking into account all these ideas, we note that the goal of the theoretical part of this paper is twofold. On the one hand, we define an average degree for infinite graphs inheriting the properties of the average degree for finite graphs. On the other hand, we generalize the function $d(p)$ for finite and infinite graphs and we prove relationships between both functions.

The next section deals with the generalization of the average degree for infinite graphs. The increasing concentric sequences are defined and the concept of the averagemeasurable graph is introduced. Besides, three infinite families of average-measurable graphs are presented.

The following section is devoted to extending the extremal function $\operatorname{ex}\left(n ; T K_{p}\right)$. The function $d_{\infty}(p)$ is defined and bounded by the corresponding 'finite version'. Some other theoretical results are proved, and they provide us with exact values for $d_{\infty}(p)$ when $1 \leq p \leq 5$.

Finally, we apply the studied concepts to the transportation of dangerous goods, paying special attention to complex networks and percolation. The paper concludes with a brief summary.

\section{Average-measurable graphs}

In this section, we define the average degree for a family of infinite graphs that we call average-measurable. We start with a sequence of finite graphs, and the average degree for infinite graphs will inherit the properties of the average degree for the finite case (see Barooah and Hespanha, 2008; Wierman and Naor, 2005; Zemanian, 1988). We are about to prove that trees are examples of average-measurable graphs. On the other hand, we introduce another family of graphs, called quasi-finite graphs, that are also proved to be average-measurable. To achieve these goals we need some notation and definitions.

Definition 1. Let $G$ be an infinite, locally finite graph, and let $\left\{G_{n}\right\}_{n \in \mathbb{N}}$ be a sequence of finite subgraphs of 
$G$. We say that $\left\{G_{n}\right\}_{n \in \mathbb{N}}$ is an increasing concentric sequence (ICS) of $G$ if the following three conditions are satisfied:

- $G_{n} \subset G_{n+1}$ for all $n$,

- $\partial G_{n} \cap \partial G_{n+1}=\emptyset$ for all $n$,

- $\cup_{n \in \mathbb{N}} G_{n}=G$,

where $\partial G_{n}=\left\langle V\left(G_{n}\right)-V\left(G_{n-1}\right)\right\rangle_{G}$ denotes the boundary of $G_{n}$.

We note that, given an infinite graph $G$ and $v \in G$, it is always possible to find an ICS. In fact, if we consider the subgraphs $G_{n}(v)=\langle\{u \in V(G): d(u, v) \leq n\}\rangle_{G}$, where $d(u, v)$ denotes the distance between the vertices $u$ and $v$, it is easy to prove that the sequence $\left\{G_{n}(v)\right\}_{n \in \mathbb{N}}$ satisfies the conditions described above for being an ICS of $G$.

Definition 2. Given an infinite, locally finite graph $G$, we define the inferior-average degree of $G$ as

$$
\underline{d}_{\infty}(G)=\inf \left\{\liminf _{n \rightarrow+\infty} d\left(G_{n}(v)\right): v \in G\right\},
$$

where $d\left(G_{n}\right)$ is the average degree of each finite graph $G_{n}(v)$.

On the other hand, we define the superior-average degree of $G$ as

$$
\bar{d}_{\infty}(G)=\sup \left\{\limsup _{n \rightarrow+\infty} d\left(G_{n}(v)\right): v \in G\right\} .
$$

Definition 3. Let $G$ be an infinite, locally finite graph. $G$ is said to be average-measurable graph if $\underline{d}_{\infty}(G)=\bar{d}_{\infty}(G)<+\infty$. Besides, in this case, we define the average degree of $G$ as $d_{\infty}(G)=\underline{d}_{\infty}(G)=\bar{d}_{\infty}(G)$.

Example 1. Let $H$ be the tree shown in Fig. 1, where the vertex $u$ is a root and the degree of the vertices of each level equals its predecessor plus one (two for the first and second levels).

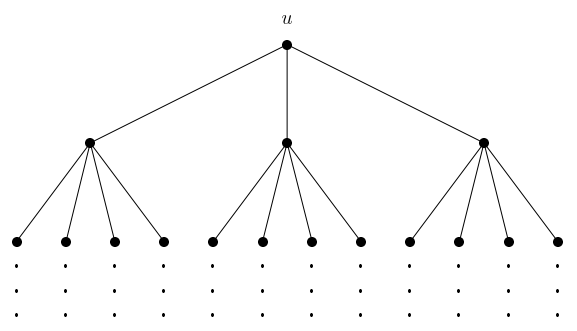

Fig. 1. Tree $H$ with an increasing degree.

If we consider the ICS $\left\{H_{n}(u)\right\}_{n \in \mathbb{N}}$, it is easy to check that, for every positive integer $n$,

$$
\left|V\left(H_{n}(u)\right)\right|=\frac{2 !+\cdots+(n+2) !}{2} .
$$

On the other hand, taking into account that each finite subgraph $H_{n}(u)$ of $\mathrm{H}$ is a tree,

$$
\left|E\left(H_{n}(u)\right)\right|=\left|V\left(H_{n}(u)\right)\right|-1,
$$

and, therefore,

$\lim _{n \rightarrow+\infty} 2 \cdot \frac{\left|E\left(H_{n}(u)\right)\right|}{\left|V\left(H_{n}(u)\right)\right|}=2 \lim _{n \rightarrow+\infty} 1-\frac{1}{\left|V\left(H_{n}(u)\right)\right|}=2$.

However, this property (proved for the vertex $u$ ) is, in fact, true for every vertex of $H$. Moreover, this property is true for every tree.

Theorem 1. Every infinite, locally finite tree $T$ is averagemeasurable and $d_{\infty}(T)=2$.

The following example shows a non-average-measurable graph.

Example 2. Let us consider the graph $G$ (see Fig. 2), obtained from the graph $H$ in the previous example and satisfying

and

$$
\partial G_{1}(v)=K_{3}
$$

$$
\left|E\left(\partial G_{n}(v)\right)\right|=n\left|V\left(G_{n}(v)\right)\right| \quad \text { for } n \geq 2 .
$$

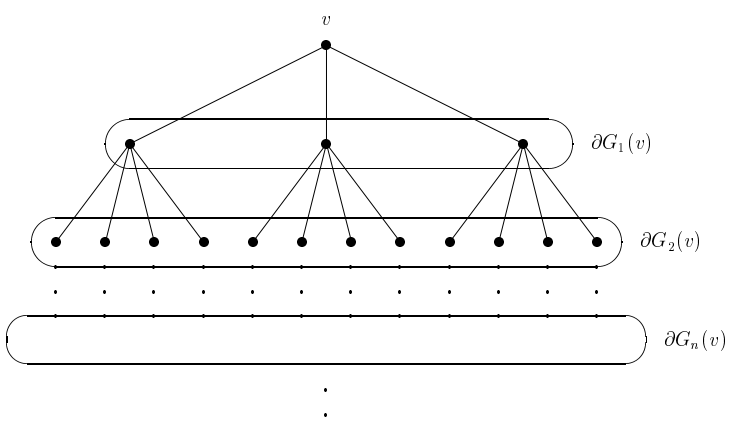

Fig. 2. Graph $G$ obtained from $H$.

By induction, it is easy to see that

$$
n\left|V\left(G_{n}(v)\right)\right| \leq\left(\begin{array}{c}
\left|V\left(\partial G_{n}(v)\right)\right| \\
2
\end{array}\right)
$$

and, therefore, it is possible to produce such a graph $G$.

On the other hand, from the construction of $G$,

$$
\left|V\left(G_{n}(v)\right)\right|=\frac{2 !+\cdots+(n+2) !}{2}
$$

and

$$
\begin{aligned}
\left|E\left(G_{n}(v)\right)\right|= & \left|V\left(G_{n}(v)\right)\right|-1+3+\cdots \\
& +2\left|V\left(G_{2}(v)\right)\right|+\cdots+n\left|V\left(G_{n}(v)\right)\right| .
\end{aligned}
$$

Now, let $M$ be the graph designed in such a way that

$$
V(M)=V(H) \cup V(G) \cup\{w\}
$$




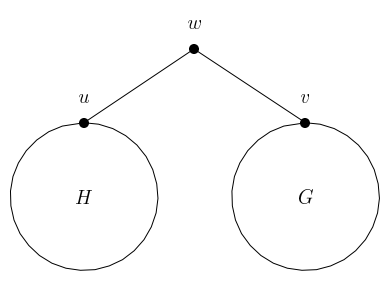

Fig. 3. Graph $M$ obtained from $H$ and $G$.

and

$$
E(M)=E(H) \cup E(G) \cup\{(u, w),(v, w)\}
$$

(see Fig. 3).

We are going to study the sequences $\left\{M_{n}(u)\right\}_{n \in \mathbb{N}}$ and $\left\{M_{n}(v)\right\}_{n \in \mathbb{N}}$. By the definition of $M_{n}(u)$, for $n \geq 2$,

$$
\left|V\left(M_{n}(u)\right)\right|=\left|V\left(H_{n}(u)\right)\right|+\left|V\left(G_{n-2}(v)\right)\right|+1
$$

and

$$
\left|E\left(M_{n}(u)\right)\right|=\left|E\left(H_{n}(u)\right)\right|+\left|E\left(G_{n-2}(v)\right)\right|+2 .
$$

To determine the limit of the sequence $\left\{d\left(M_{n}(u)\right)\right\}_{n \geq 2}$, it is sufficient to analyze the behavior of the quotient $\left|E\left(M_{n}(u)\right)\right| /\left|V\left(M_{n}(u)\right)\right|$. By applying the well-known Stolz theorem for sequences

$$
\begin{aligned}
& \frac{\left|E\left(M_{n+1}(u)\right)\right|-\left|E\left(M_{n}(u)\right)\right|}{\left|V\left(M_{n+1}(u)\right)\right|-\left|V\left(M_{n}(u)\right)\right|} \\
& =\frac{\frac{(n+3) !}{2}+\frac{(n+1) !}{2}+(n-1) \cdot \frac{2 !+\cdots+(n+1) !}{2}}{\frac{(n+3) !}{2}+\frac{(n+1) !}{2}} \\
& =1+\frac{(n-1) \cdot \frac{2 !+\cdots+(n+1) !}{2}}{\frac{(n+3) !}{2}+\frac{(n+1) !}{2}} .
\end{aligned}
$$

If we apply again the Stolz theorem, we get

$$
\lim _{n \rightarrow+\infty} \frac{(n-1)(2 !+\cdots+(n+1) !)}{(n+3) !+(n+1) !}=0 .
$$

It follows that

$$
\lim _{n \rightarrow+\infty} 2 \frac{\left|E\left(M_{n}(u)\right)\right|}{\left|V\left(M_{n}(u)\right)\right|}=2
$$

and, therefore, $\underline{d}_{\infty}(M) \leq 2$.

Now we analyze what happens with the sequence $\left\{M_{n}(v)\right\}_{n \in \mathbb{N}}$ :

$$
\left|V\left(M_{n}(v)\right)\right|=\left|V\left(H_{n-2}(u)\right)\right|+\left|V\left(G_{n}(v)\right)\right|+1
$$

and

$$
\left|E\left(M_{n}(v)\right)\right|=\left|E\left(H_{n-2}(u)\right)\right|+\left|E\left(G_{n}(v)\right)\right|+2 .
$$

We apply the Stolz theorem to compute the limit of the average degree of each subgraph $M_{n}(v)$ :

$$
\begin{aligned}
\frac{\left|E\left(M_{n+1}(v)\right)\right|-\left|E\left(M_{n}(v)\right)\right|}{\left|V\left(M_{n+1}(v)\right)\right|-\left|V\left(M_{n}(v)\right)\right|} & \\
& =1+\frac{(n+1) \cdot \frac{2 !+\cdots+(n+3) !}{2}}{\frac{(n+3) !}{2}+\frac{(n+1) !}{2}} .
\end{aligned}
$$

By applying the Stolz theorem again,

$$
\lim _{n \rightarrow+\infty} \frac{(n+1)(2 !+\cdots+(n+3) !)}{(n+3) !+(n+1) !}=+\infty .
$$

Hence

$$
\lim _{n \rightarrow+\infty} 2 \cdot \frac{\left|E\left(M_{n}(u)\right)\right|}{\left|V\left(M_{n}(u)\right)\right|}=+\infty
$$

and, therefore, $\bar{d}_{\infty}(M)=+\infty$. Thus,

$$
\underline{d}_{\infty}(M) \leq 2<\bar{d}_{\infty}(M)=+\infty,
$$

and $M$ is non-average-measurable.

2.1. Quasi-finite graphs. Next we define a family of infinite graphs which are average-measurable when their maximal degree is bounded.

Definition 4. Let $G$ be an infinite, locally finite graph. The ICS $\left\{G_{n}\right\}_{n \in \mathbb{N}}$ satisfies the so-called boundary condition when

$$
\lim _{n \rightarrow+\infty} \frac{\left|V\left(\partial G_{n}\right)\right|}{\left|V\left(G_{n}\right)\right|}=0 .
$$

Remark 1. The boundary condition

$$
\lim _{n \rightarrow+\infty} \frac{\left|V\left(\partial G_{n}\right)\right|}{\left|V\left(G_{n}\right)\right|}=0
$$

is equivalent to

$$
\lim _{n \rightarrow+\infty} \frac{\left|V\left(G_{n-1}\right)\right|}{\left|V\left(G_{n}\right)\right|}=1,
$$

since

$$
\begin{aligned}
\frac{\left|V\left(\partial G_{n}\right)\right|}{\left|V\left(G_{n}\right)\right|} & =\frac{\left|V\left(G_{n}\right)\right|-\left|V\left(G_{n-1}\right)\right|}{\left|V\left(G_{n}\right)\right|} \\
& =1-\frac{\left|V\left(G_{n-1}\right)\right|}{\left|V\left(G_{n}\right)\right|} .
\end{aligned}
$$

Definition 5. Let $G$ be an infinite, locally finite graph. $G$ is said to be quasi-finite if there exists a vertex $v \in G$ such that the ICS $\left\{G_{n}(v)\right\}_{n \in \mathbb{N}}$ satisfies the boundary condition.

The following result shows that the previous definition does not depend on the chosen vertex, i.e., if there exists a vertex $v$ for which $\left\{G_{n}(v)\right\}_{n \in \mathbb{N}}$ satisfies the boundary condition, then it is satisfied for all $v$. 
Lemma 1. Let $G$ be an infinite, locally finite graph. If $G$ is quasi-finite, then the sequence $\left\{G_{n}(v)\right\}_{n \in \mathbb{N}}$ satisfies the boundary condition for all $v \in G$, i.e.,

$$
\lim _{n \rightarrow+\infty} \frac{\left|V\left(\partial G_{n}(v)\right)\right|}{\left|V\left(G_{n}(v)\right)\right|}=0 .
$$

Proof. Let $G$ be a quasi-finite graph and $v_{0}$ be a vertex such that the ICS $\left\{G_{n}\left(v_{0}\right)\right\}_{n \in \mathbb{N}}$ satisfies the boundary condition. Given $v \in G$, we denote by $r$ the distance between $v$ and $v_{0}\left(r=d\left(v, v_{0}\right)\right)$. Taking into account Remark 1] it is sufficient to prove the equality

$$
\lim _{n \rightarrow+\infty} \frac{\left|V\left(G_{n-1}(v)\right)\right|}{\left|V\left(G_{n}(v)\right)\right|}=1
$$

to show that the boundary condition is satisfies.

Consequently, we note that, for $n \geq r+1$,

$$
\begin{gathered}
G_{n-r-1}\left(v_{0}\right) \subseteq G_{n-1}(v), \\
G_{n}(v) \subseteq G_{n+r}\left(v_{0}\right) .
\end{gathered}
$$

Hence

$$
\frac{\left|V\left(G_{n-1}(v)\right)\right|}{\left|V\left(G_{n}(v)\right)\right|} \geq \frac{\left|V\left(G_{n-r-1}\left(v_{0}\right)\right)\right|}{\left|V\left(G_{n+r}\left(v_{0}\right)\right)\right|} .
$$

Furthermore,

$$
\begin{aligned}
& \frac{\left|V\left(G_{n-r-1}\left(v_{0}\right)\right)\right|}{\left|V\left(G_{n+r}\left(v_{0}\right)\right)\right|} \\
& =\frac{\left|V\left(G_{n-r-1}\left(v_{0}\right)\right)\right|}{\left|V\left(G_{n-r}\left(v_{0}\right)\right)\right|} \frac{\left|V\left(G_{n-r}\left(v_{0}\right)\right)\right|}{\left|V\left(G_{n-r+1}\left(v_{0}\right)\right)\right|} \\
& \cdots \frac{\left|V\left(G_{n+r-1}\left(v_{0}\right)\right)\right|}{\left|V\left(G_{n+r}\left(v_{0}\right)\right)\right|},
\end{aligned}
$$

but

Finally,

$$
\begin{array}{ccc}
\frac{\left|V\left(G_{n-r-1}\left(v_{0}\right)\right)\right|}{\left|V\left(G_{n-r}\left(v_{0}\right)\right)\right|} \quad & & \\
\vdots & & \\
\frac{\left|V\left(G_{n+r-1}\left(v_{0}\right)\right)\right|}{\left|V\left(G_{n+r}\left(v_{0}\right)\right)\right|} & & \\
n \rightarrow+\infty & 1 .
\end{array}
$$

$$
\begin{aligned}
1 & \geq \lim _{n \rightarrow+\infty} \frac{\left|V\left(G_{n-1}(v)\right)\right|}{\left|V\left(G_{n}(v)\right)\right|} \\
& \geq \lim _{n \rightarrow+\infty} \frac{\left|V\left(G_{n-r-1}\left(v_{0}\right)\right)\right|}{\left|V\left(G_{n+r}\left(v_{0}\right)\right)\right|}=1 .
\end{aligned}
$$

Thus,

$$
\lim _{n \rightarrow+\infty} \frac{\left|V\left(G_{n-1}(v)\right)\right|}{\left|V\left(G_{n}(v)\right)\right|}=1,
$$

and the result follows.

Remark 2. By reasoning as in Lemma 1 we get

$$
\lim _{n \rightarrow+\infty} \frac{\left|V\left(G_{n}(v)\right)\right|}{\left|V\left(G_{n+k}(v)\right)\right|}=1
$$

for all positive integer $k$.
The previous lemma can be generalized to other sequences. In fact, given a finite $G_{0} \subset G$, we consider the ICS $\left\{G_{n}\left(G_{0}\right)\right\}_{n \geq 0}$ as the sequence defined as follows:

$$
G_{n}\left(G_{0}\right)=\left\langle\left\{u \in V(G): d\left(u, G_{0}\right) \leq n\right\}\right\rangle_{G} .
$$

Theorem 2. Let $G$ be an infinite, locally finite graph. Then $G$ is quasi-finite if and only if there exists a finite subgraph $G_{0} \subset G$ satisfying the boundary condition, that is to say,

$$
\lim _{n \rightarrow+\infty} \frac{\left|V\left(\partial G_{n}\left(G_{0}\right)\right)\right|}{\left|V\left(G_{n}\left(G_{0}\right)\right)\right|}=0 .
$$

Besides, if the aforementioned assertion is true, then the boundary condition is satisfied for every finite subgraph $G_{0} \subset G$.

Proof. Let $G$ be an infinite, quasi-finite graph, and $G_{0} \subset$ $G$ be a finite subgraph. We are proving that

$$
\lim _{n \rightarrow+\infty} \frac{\left|V\left(G_{n-1}\left(G_{0}\right)\right)\right|}{\left|V\left(G_{n}\left(G_{0}\right)\right)\right|}=1 .
$$

For this purpose, let us consider $v_{0} \in V\left(G_{0}\right)$ and denote by $r$ the diameter of $G_{0}\left(r=\operatorname{diam}\left(G_{0}\right)\right)$. Consequently,

$$
\begin{gathered}
G_{n-1}\left(v_{0}\right) \subseteq G_{n-1}\left(G_{0}\right) \\
G_{n}\left(G_{0}\right) \subseteq G_{n+r}\left(v_{0}\right)
\end{gathered}
$$

for all $n$. It follows that

$$
\frac{\left|V\left(G_{n-1}\left(G_{0}\right)\right)\right|}{\left|V\left(G_{n}\left(G_{0}\right)\right)\right|} \geq \frac{\left|V\left(G_{n-1}\left(v_{0}\right)\right)\right|}{\left|V\left(G_{n+r}\left(v_{0}\right)\right)\right|} .
$$

Bearing in mind the inequality

$$
\frac{\left|V\left(G_{n-1}\left(G_{0}\right)\right)\right|}{\left|V\left(G_{n}\left(G_{0}\right)\right)\right|} \leq 1
$$

and by applying Remark 2 .

$$
\begin{aligned}
1 & \geq \lim _{n \rightarrow+\infty} \frac{\left|V\left(G_{n-1}\left(G_{0}\right)\right)\right|}{\left|V\left(G_{n}\left(G_{0}\right)\right)\right|} \\
& \geq \lim _{n \rightarrow+\infty} \frac{\left|V\left(G_{n-1}\left(v_{0}\right)\right)\right|}{\left|V\left(G_{n+r}\left(v_{0}\right)\right)\right|}=1 .
\end{aligned}
$$

Therefore,

$$
\lim _{n \rightarrow+\infty} \frac{\left|V\left(G_{n-1}\left(G_{0}\right)\right)\right|}{\left|V\left(G_{n}\left(G_{0}\right)\right)\right|}=1 .
$$

In order to prove the converse implication, we suppose that there exists a $G_{0}$ such that the sequence $\left\{G_{n}\left(G_{0}\right)\right\}$ satisfies the boundary condition. Since that $v_{0} \in V\left(G_{0}\right)$ and $r=\operatorname{diam}\left(G_{0}\right)$,

$$
G_{n-r-1}\left(G_{0}\right) \subseteq G_{n-1}\left(v_{0}\right)
$$

and

$$
G_{n}\left(v_{0}\right) \subseteq G_{n}\left(G_{0}\right)
$$


Hence

$$
\frac{\left|V\left(G_{n-1}\left(v_{0}\right)\right)\right|}{\left|V\left(G_{n}\left(v_{0}\right)\right)\right|} \geq \frac{\left|V\left(G_{n-r-1}\left(G_{0}\right)\right)\right|}{\left|V\left(G_{n}\left(v_{0}\right)\right)\right|} .
$$

By reasoning as in Remark2, we see that

$$
\lim _{n \rightarrow+\infty} \frac{\left|V\left(G_{n-r-1}\left(G_{0}\right)\right)\right|}{\left|V\left(G_{n}\left(G_{0}\right)\right)\right|}=1,
$$

so that

$$
\lim _{n \rightarrow+\infty} \frac{\left|V\left(G_{n-1}\left(v_{0}\right)\right)\right|}{\left|V\left(G_{n}\left(v_{0}\right)\right)\right|}=1
$$

Now, we are going to prove that every quasi-finite graph with a bounded maximal degree is average-measurable.

Theorem 3. Let $G$ be an infinite, locally finite graph with $\Delta(G)<+\infty$. If $G$ is quasi-finite, then $G$ is averagemeasurable.

Proof. Let $G$ be an infinite graph with maximal degree $\Delta(G)=\Delta<+\infty$. Given $v \in G$, we consider the sequence $G_{n}=G_{n}(v)$ for $n \in \mathbb{N}$. Since $\Delta(G)<+\infty$, we know that $d\left(G_{n}\right) \leq 2 \Delta$. To prove that $\left\{d\left(G_{n}\right)\right\}$ is convergent, we check that, in fact, it is a Cauchy sequence, that is,

$$
\lim _{n \rightarrow+\infty}\left|d\left(G_{n+1}\right)-d\left(G_{n}\right)\right|=0 .
$$
follows:

$$
\begin{aligned}
s_{n} & =\left|\frac{\left|E\left(G_{n+1}\right)\right|}{\left|V\left(G_{n+1}\right)\right|}-\frac{\left|E\left(G_{n}\right)\right|}{\left|V\left(G_{n}\right)\right|}\right| \\
& =\left|\frac{\left|E\left(G_{n+1}\right)\right| \cdot\left|V\left(G_{n}\right)\right|-\left|E\left(G_{n}\right)\right| \cdot\left|V\left(G_{n+1}\right)\right|}{\left|V\left(G_{n}\right)\right| \cdot\left|V\left(G_{n+1}\right)\right|}\right| .
\end{aligned}
$$

If we set

$$
\begin{aligned}
E\left(\partial G_{n}, \partial G_{n+1}\right)=\{ & \left(w_{n}, w_{n+1}\right) \in E(G): \\
& \left.w_{n} \in \partial G_{n}, w_{n+1} \in \partial G_{n+1}\right\},
\end{aligned}
$$

then (see Fig. 4)

$$
\begin{aligned}
\left|E\left(G_{n+1}\right)\right|= & \left|E\left(G_{n}\right)\right|+\left|E\left(\partial G_{n}, \partial G_{n+1}\right)\right| \\
& +\left|E\left(\partial G_{n+1}\right)\right| .
\end{aligned}
$$

Thus,

$$
\begin{aligned}
s_{n}= & \mid \frac{1}{\left|V\left(G_{n}\right)\right| \cdot\left|V\left(G_{n+1}\right)\right|} \\
& {\left[| V ( G _ { n } ) | \left(\left|E\left(G_{n}\right)\right|+\left|E\left(\partial G_{n}, \partial G_{n+1}\right)\right|\right.\right.} \\
& \left.\left.+\left|E\left(\partial G_{n+1}\right)\right|\right)-\cdots-\left|E\left(G_{n}\right)\right| \cdot\left|V\left(G_{n+1}\right)\right|\right] \mid \\
\leq & \frac{\left|E\left(G_{n}\right)\right||| V\left(G_{n}\right)|-| V\left(G_{n+1}\right)||}{\left|V\left(G_{n}\right)\right| \cdot\left|V\left(G_{n+1}\right)\right|} \\
& +\frac{\left|E\left(\partial G_{n}, \partial G_{n+1}\right)\right|}{\left|V\left(G_{n+1}\right)\right|}+\cdots+\frac{\left|E\left(\partial G_{n+1}\right)\right|}{\left|V\left(G_{n+1}\right)\right|} .
\end{aligned}
$$

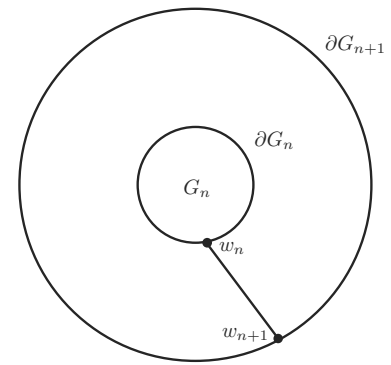

Fig. 4. Edge decomposition $E\left(G_{n+1}\right)$.

However,

$\frac{\left|E\left(G_{n}\right)\right||| V\left(G_{n}\right)|-| V\left(G_{n+1}\right)||}{\left|V\left(G_{n}\right)\right| \cdot\left|V\left(G_{n+1}\right)\right|} \leq \Delta \cdot \frac{\left|V\left(\partial G_{n+1}\right)\right|}{\left|V\left(G_{n+1}\right)\right|}$,

$$
\frac{\left|E\left(\partial G_{n}, \partial G_{n+1}\right)\right|}{\left|V\left(G_{n+1}\right)\right|} \leq \frac{\Delta \cdot\left|V\left(\partial G_{n}\right)\right|}{\left|V\left(G_{n+1}\right)\right|} \leq \Delta \cdot \frac{\left|V\left(\partial G_{n}\right)\right|}{\left|V\left(G_{n}\right)\right|},
$$

and

$$
\frac{\left|E\left(\partial G_{n+1}\right)\right|}{\left|V\left(G_{n+1}\right)\right|} \leq \Delta \cdot \frac{\left|V\left(\partial G_{n+1}\right)\right|}{\left|V\left(G_{n+1}\right)\right|} .
$$

Hence

$$
\begin{aligned}
s_{n} \leq & \Delta \cdot \frac{\left|V\left(\partial G_{n+1}\right)\right|}{\left|V\left(G_{n+1}\right)\right|}+\Delta \cdot \frac{\left|V\left(\partial G_{n}\right)\right|}{\left|V\left(G_{n}\right)\right|} \\
& +\Delta \cdot \frac{\left|V\left(\partial G_{n+1}\right)\right|}{\left|V\left(G_{n+1}\right)\right|} .
\end{aligned}
$$

As

$$
\lim _{n \rightarrow+\infty} \frac{\left|V\left(\partial G_{n}\right)\right|}{\mid V\left(G_{n} \mid\right.}=0
$$

we get

$$
\lim _{n \rightarrow+\infty} s_{n}=0
$$

and, therefore, the sequence $\left\{d\left(G_{n}\right)\right\}_{n \in \mathbb{N}}$ is convergent.

Finally, to reach $\underline{d}_{\infty}(G)=\bar{d}_{\infty}(G)$, we are going to prove that, for all $u \in V(G)$,

$$
\lim _{n \rightarrow+\infty} \frac{\left|E\left(G_{n}(u)\right)\right|}{\mid V\left(G_{n}(u) \mid\right.}=\lim _{n \rightarrow+\infty} \frac{\left|E\left(G_{n}(v)\right)\right|}{\mid V\left(G_{n}(v) \mid\right.}=t .
$$

For this purpose, we consider $u \in V(G)$ and $r=d_{G}(u, v)$. Accordingly,

$$
\begin{aligned}
& t_{n} \\
& =\left|\frac{\left|E\left(G_{n}(u)\right)\right|}{\left|V\left(G_{n}(u)\right)\right|}-\frac{\mid E\left(G_{n}(v) \mid\right.}{\mid V\left(G_{n}(v) \mid\right.}\right| \\
& =\left|\frac{\left|E\left(G_{n}(u)\right)\right|\left|V\left(G_{n}(v)\right)\right|-\left|E\left(G_{n}(v)\right)\right|\left|V\left(G_{n}(u)\right)\right|}{\left|V\left(G_{n}(u)\right)\right|\left|V\left(G_{n}(v)\right)\right|}\right| .
\end{aligned}
$$

Besides, taking into account that

$$
G_{n-r}(v) \subseteq G_{n}(u) \subseteq G_{n+r}(v)
$$


for $n \geq r$, we have that

$$
\begin{aligned}
t_{n} \leq \mid & \frac{1}{\left|V\left(G_{n-r}(v)\right)\right|\left|V\left(G_{n}(v)\right)\right|} \\
& \times\left(\left|E\left(G_{n+r}(v)\right)\right|\left|V\left(G_{n}(v)\right)\right|\right. \\
& \left.-\left|E\left(G_{n}(v)\right)\right|\left|V\left(G_{n-r}(v)\right)\right|\right) \mid \\
& \times\left|\frac{\left|E\left(G_{n+r}(v)\right)\right|}{\left|V\left(G_{n-r}(v)\right)\right|}-\frac{\left|E\left(G_{n}(v)\right)\right|}{\left|V\left(G_{n}(v)\right)\right|}\right| .
\end{aligned}
$$

Since

$$
\lim _{n \rightarrow+\infty} \frac{\left|V\left(G_{n}(v)\right)\right|}{\left|V\left(G_{n+1}(v)\right)\right|}=1
$$

and

$$
\begin{aligned}
\frac{\left|E\left(G_{n+r}(v)\right)\right|}{\left|V\left(G_{n-r}(v)\right)\right|}= & \frac{\left|E\left(G_{n+r}(v)\right)\right|}{\left|V\left(G_{n+r}(v)\right)\right|} \frac{\left|V\left(G_{n+r}(v)\right)\right|}{\left|V\left(G_{n+r-1}(v)\right)\right|} \\
& \times \frac{\left|V\left(G_{n-r+1}(v)\right)\right|}{\left|V\left(G_{n-r}(v)\right)\right|}
\end{aligned}
$$

we conclude that

$$
\lim _{n \rightarrow+\infty} \frac{\left|E\left(G_{n+r}(v)\right)\right|}{\left|V\left(G_{n-r}(v)\right)\right|}=t
$$

and, therefore, $\lim _{n \rightarrow+\infty} t_{n}=0$.

Now, we note that the condition $\Delta(G)<+\infty$ is necessary in this theorem, as we can see with the graph $G$ from Fig. 5. $G$ is quasi-finite but not average-measurable.

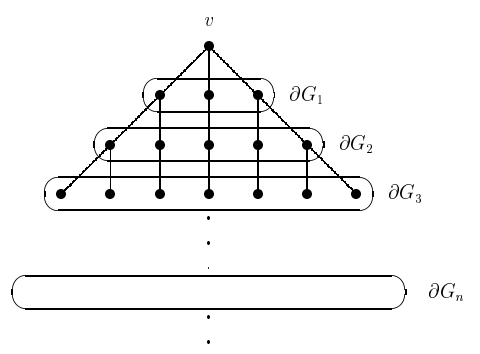

Fig. 5. Quasi-finite graph with $\Delta(G)=+\infty$, but non-averagemeasurable.

This graph is defined in such a way that the subgraph $\partial G_{n}$ is a complete graph of size $2 n+1$, for $n \geq 1$. Thus,

$$
\left|V\left(G_{n}(v)\right)\right|=1+3+5+\cdots+(2 n+1)
$$

and

$$
\begin{aligned}
\left|E\left(G_{n}(v)\right)\right|= & 3+5+\cdots+(2 n+1) \\
& +\left(\begin{array}{l}
3 \\
2
\end{array}\right)+\left(\begin{array}{l}
5 \\
2
\end{array}\right)+\cdots \\
& +\left(\begin{array}{c}
2 n+1 \\
2
\end{array}\right) .
\end{aligned}
$$

$G$ is quasi-finite because

$$
\begin{aligned}
\lim _{n \rightarrow+\infty} \frac{\left|V\left(\partial G_{n}(v)\right)\right|}{\left|V\left(G_{n}(v)\right)\right|} \\
\quad=\lim _{n \rightarrow+\infty} \frac{2 n+1}{1+3+5+\cdots+(2 n+1)}=0 .
\end{aligned}
$$

In order to get the limit of $\left|E\left(G_{n}(v)\right)\right| /\left|V\left(G_{n}(v)\right)\right|$, we apply the Stolz theorem:

$$
\begin{aligned}
& \frac{\left|E\left(G_{n+1}(v)\right)\right|-\left|E\left(G_{n}(v)\right)\right|}{\left|V\left(G_{n+1}(v)\right)\right|-\left|V\left(G_{n}(v)\right)\right|} \\
& \quad=\frac{2 n+3+\left(\begin{array}{c}
2 n+3 \\
2
\end{array}\right)}{2 n+3}=+\infty .
\end{aligned}
$$

Thus, $\bar{d}_{\infty}(G)=+\infty$ and $G$ is not average-measurable.

On the other hand, we consider the graph $H$ from Example 1 to find an average-measurable graph which is not quasi-finite. Actually, since $H$ is a tree, this graph is average-measurable; however, it is not quasi-finite:

$$
\begin{aligned}
\frac{\left|V\left(\partial H_{n}(u)\right)\right|}{\left|V\left(H_{n}(u)\right)\right|} & =\frac{\left|V\left(H_{n}(u)\right)\right|-\left|V\left(H_{n-1}(u)\right)\right|}{\left|V\left(H_{n}(u)\right)\right|} \\
& =\frac{(n+2) !}{2 !+\cdots+(n+2) !} .
\end{aligned}
$$

By applying the Stolz theorem,

$$
\lim _{n \rightarrow+\infty} \frac{(n+3) !-(n+2) !}{(n+3) !}=\lim _{n \rightarrow+\infty} \frac{n+2}{n+3}=1 \neq 0
$$

and, therefore, the sequence $\left\{H_{n}(u)\right\}$ does not satisfy the boundary condition, and hence $H$ is not quasi-finite.

2.2. Periodic graphs. Now, we are going to show that periodic graphs are quasi-finite. These graphs are very useful because they are frequent and easily computed. We can find examples of periodic graphs in tiling and patterns (Grünbaum and Shephard, 1987) or Cayley diagrams (Cayley, 1895; Frucht, 1938), and they even appear as the resultant graphs of solving linear systems (Bauderon, 1989).

Here we recall some prior results on periodic graphs. We denote by $\mathbf{C}$ the unit square $[0,1] \times[0,1] \subset \mathbb{R}^{2}$, and we define a cellular graph as the graph satisfying $V(G) \subset$ $\mathrm{C}$ with no isolated vertices. Thus, given a cellular graph $G$, we define the 2-dimensional periodic graph $\left(\mathcal{M}_{G}\right)$ as the graph obtained from $G$ as follows:

$$
\begin{gathered}
V\left(\mathcal{M}_{G}\right)=\left\{\tau_{(m, n)}(v): v \in V(G) \text { and }(m, n) \in \mathbb{Z}^{2}\right\}, \\
E\left(\mathcal{M}_{G}\right)=\left\{\left(\tau_{(m, n)}(u), \tau_{(m, n)}(v)\right):\right. \\
\left.(u, v) \in E(G) \text { and }(m, n) \in \mathbb{Z}^{2}\right\},
\end{gathered}
$$


where $\tau_{(m, n)}$ denotes the translation by vector $(m, n)$ in the plane.

If $\mathcal{M}_{G}$ is a 2-periodic graph generated by the cellular graph $G$, then we define the 8-neighbors of $G$ as the subgraphs $\tau_{(i, j)}(G)$ of $\mathcal{M}_{G}$ such that $i \in\{-1,0,1\}$ and $j \in\{-1,0,1\}$, with $(i, j) \neq(0,0)$.

Given a cellular graph $G$ and the 2-periodic graph generated by $G, \mathcal{M}_{G}$, we define the $n$-square of center $G$ and radius $n\left(\prod_{n} G\right)$ as the subgraph of $\mathcal{M}_{G}$ :

$\prod_{n} G=\left\{\tau_{(i, j)}(G):(i, j) \in \mathbb{Z}^{2}, \max \{|i|,|j|\} \leq n\right\}$.

We are proving that, in fact, the 2-periodic graphs are quasi-finite and average-measurable (since they have a bounded maximal degree).

Theorem 4. Every infinite, periodic, connected graph $\mathcal{M}_{G}$ generated by the cellular graph $G$ is quasi-finite and average-measurable.

Proof. Let $G$ be a cellular graph and $\mathcal{M}=\mathcal{M}_{G}$ be the 2-periodic graph generated from $G$. By Theorem 2 it is sufficient to prove that

$$
\lim _{n \rightarrow+\infty} \frac{\left|V\left(\partial \mathcal{M}_{n}(G)\right)\right|}{\left|V\left(\mathcal{M}_{n}(G)\right)\right|}=0
$$

to show that $\mathcal{M}$ is quasi-finite. We recall that

$$
\mathcal{M}_{n}(G)=\left\langle u \in V(\mathcal{M}): d(u, G)_{\mathcal{M}} \leq n\right\rangle_{\mathcal{M}} .
$$

Consider

$$
d=\max \left\{d(u, G): u \in G_{i}, 1 \leq i \leq 8\right\},
$$

where $G_{i}$ are the 8-neighbors of $G$. Firstly, for all $n \geq d$, it is easily seen that (see Fig. 6)

$$
\prod_{\left\lfloor\frac{n}{d}\right\rfloor} G \subseteq \mathcal{M}_{n}(G) \subseteq \prod_{n} G
$$

because if $v \in \prod_{\left\lfloor\frac{n}{d}\right\rfloor} G$, then (see Fig. 7)

$$
d(u, G) \leq d+d\left(u, \prod_{\left\lfloor\frac{n}{d}-1\right\rfloor} G\right) \leq \cdots \leq d\left\lfloor\frac{n}{d}\right\rfloor \leq n .
$$

Accordingly,

$$
\begin{aligned}
& \frac{\left|V\left(\partial \mathcal{M}_{n}(G)\right)\right|}{\left|V\left(\mathcal{M}_{n}(G)\right)\right|} \\
& \quad \leq \frac{\left|V\left(\partial \mathcal{M}_{n}(G)\right)\right|}{\left|V\left(\prod_{\left\lfloor\frac{n}{d}\right\rfloor} G\right)\right|} \leq \frac{\left|V\left(\partial \mathcal{M}_{n}(G)\right)\right|}{\left(2\left\lfloor\frac{n}{d}\right\rfloor+1\right)^{2}|V(G)|} .
\end{aligned}
$$

On the other hand, for all $n \geq 1$, let us consider $k(n)=\left|V\left(\partial \mathcal{M}_{n}(G)\right)\right|$. As

$$
V\left(\mathcal{M}_{n}(G)\right)=\bigcup_{i=0}^{n} V\left(\partial \mathcal{M}_{i}(G)\right) \subseteq V\left(\prod_{n} G\right),
$$

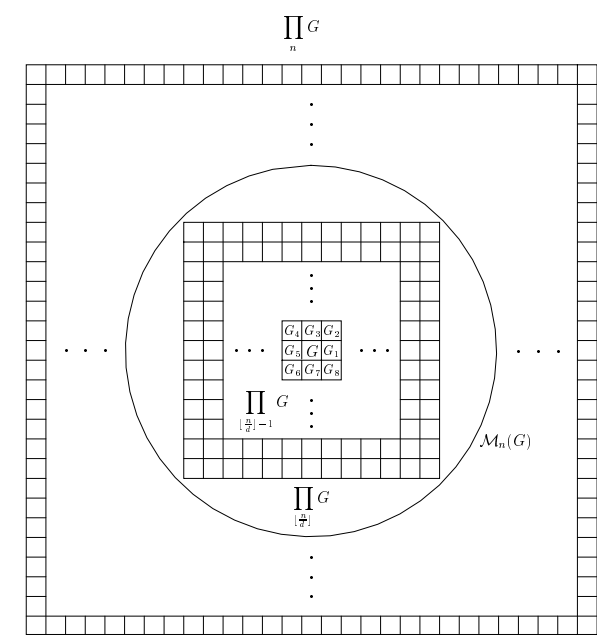

Fig. 6. Chain of inclusions for $\mathcal{M}_{n}(G)$.

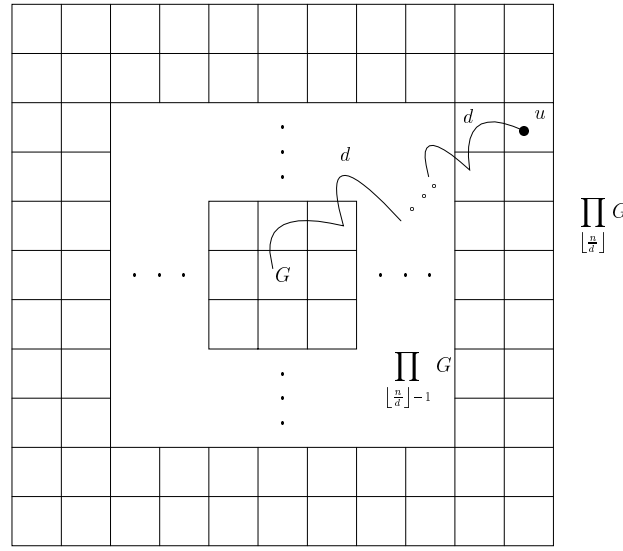

Fig. 7. $\prod_{\left\lfloor\frac{n}{d}\right\rfloor} G \subseteq \mathcal{M}_{n}(G)$.

we have

$$
s_{n}=k(1)+k(2)+\cdots+k(n) \leq(2 n+1)^{2}|V(G)| .
$$

Suppose that

$$
\limsup _{n \rightarrow+\infty} \frac{k(n)}{n^{2}}=l,
$$

with $l>0$. By applying the Stolz theorem to the quotient $s_{n} /(2 n+1)^{2}$, we get that

$$
\begin{aligned}
\frac{s_{n+1}-s_{n}}{(2 n+3)^{2}-(2 n+1)^{2}} & =\frac{k(n+1)}{8 n+9} \\
& =\frac{k(n+1)}{(n+1)^{2}} \frac{(n+1)^{2}}{8 n+9}
\end{aligned}
$$

and, therefore,

$$
\begin{aligned}
\limsup _{n \rightarrow+\infty} & \frac{s_{n}}{(2 n+1)^{2}|V(G)|} \\
& =\frac{1}{|V(G)|} \limsup _{n \rightarrow+\infty} \frac{k(n+1)}{(n+1)^{2}} \frac{(n+1)^{2}}{8 n+9}=+\infty .
\end{aligned}
$$


But this is not possible, because we were assuming that

$$
\frac{s_{n}}{(2 n+1)^{2}|V(G)|} \leq 1
$$

Consequently,

$$
\lim _{n \rightarrow+\infty} \frac{k(n)}{n^{2}}=0
$$

and, therefore,

$$
\begin{aligned}
\lim _{n \rightarrow+\infty} & \frac{\left|V\left(\partial \mathcal{M}_{n}(G)\right)\right|}{\left(2\left\lfloor\frac{n}{d}\right\rfloor+1\right)^{2}|V(G)|} \\
& \leq \frac{1}{|V(G)|} \lim _{n \rightarrow+\infty} \frac{k(n)}{n^{2}} \frac{n^{2}}{\left(2\left(\frac{n}{d}-1\right)+1\right)^{2}}=0 .
\end{aligned}
$$

Thus

$$
\lim _{n \rightarrow+\infty} \frac{\left|V\left(\partial \mathcal{M}_{n}(G)\right)\right|}{\left|V\left(\mathcal{M}_{n}(G)\right)\right|}=0
$$

and $\mathcal{M}$ is quasi-finite. Besides, since $\mathcal{M}$ is a 2-periodic graph, the assertion $\Delta(\mathcal{M})<+\infty$ is satisfied, and (by applying Theorem 3) $\mathcal{M}$ is average-measurable.

Now, we present an illustration of a 2-periodic graph.

Example 3. We consider the 2-periodic graph $\mathcal{M}$ generated by the cellular graph $G$ as in Fig. 8
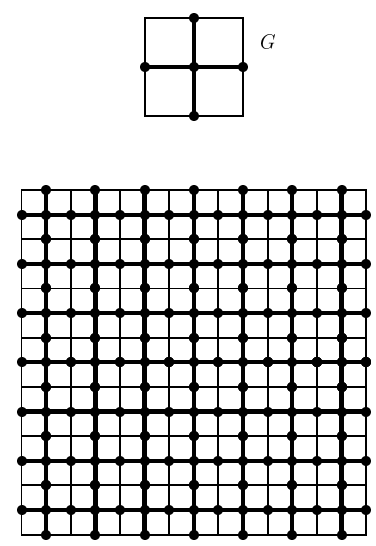

Fig. 8. Periodic graph with the average degree $\frac{8}{3}$.

Since $\mathcal{M}$ is connected, by applying Theorem 4 , we deduce that this graph is average-measurable. Let us consider the sequence $\left\{\mathcal{M}_{n}(G)\right\}$. Since the sequence $\left\{d\left(\mathcal{M}_{n}(G)\right)\right\}$ is convergent, we know that

$$
\lim _{n \rightarrow+\infty} d\left(\mathcal{M}_{n}(G)\right)=\lim _{n \rightarrow+\infty} d\left(\mathcal{M}_{2 n}(G)\right) .
$$

On the other hand,

$$
\left|V\left(\mathcal{M}_{2 n}(G)\right)\right|=5+16+6\left(4+8+\cdots+2^{n}\right)
$$

and

$$
\left|E\left(\mathcal{M}_{2 n}(G)\right)\right|=4+16+8\left(4+8+\cdots+2^{n}\right)
$$

and, therefore, by applying Stolz Theorem,

$$
\lim _{n \rightarrow+\infty} d\left(\mathcal{M}_{2 n}(G)\right)=\frac{8}{3} .
$$

Remark 3. In interconnection networks, it is important to control their behavior when edges are subdivided or contracted. Taking into account the definition of an average degree for infinite graphs that we have just introduced, it is straightforward to check that if we subdivide one or more edges in a graph, the average degree of the resulting graph is less than or equal to the original.

In the following example (Fig. 9), it is easy to check that the average degree is four. If subdivisions of each edge are performed (Figure 10), we obtain a graph with a smaller average degree (the one shown in Fig. 8).

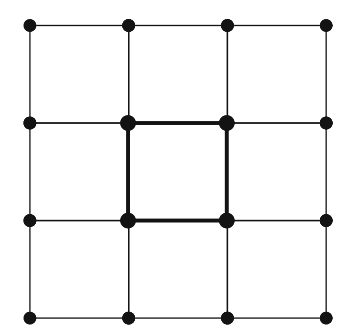

Fig. 9. Periodic graph with the average degree 4.

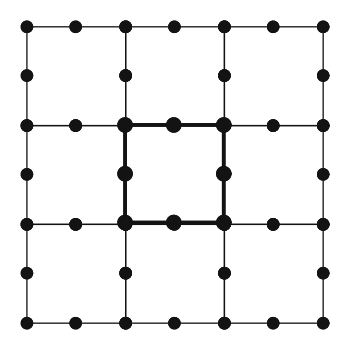

Fig. 10. Periodic graph with the average degree $\frac{8}{3}$.

In the graph displayed in Fig. 11 we can observe the behavior of the average degree when performing contractions of edges.

Another kind of periodic graph is the 1-dimensional case. This graph $\mathcal{M}_{G}^{1}$ is generated by a cellular graph $G$ and horizontal translations $G_{i}$ of the graph $G$ (see Fig. 13). Here we denote by $v_{i}$ the translated vertex of $v$ in $G_{i}$, for all integers $i$ and all $v \in V(G)$.

Now, we are able to formulate a general result.

Theorem 5. Let $G$ be a finite cellular graph. If $\mathcal{M}_{G}^{1}$ is connected, then it is quasi-finite and, therefore, averagemeasurable. 


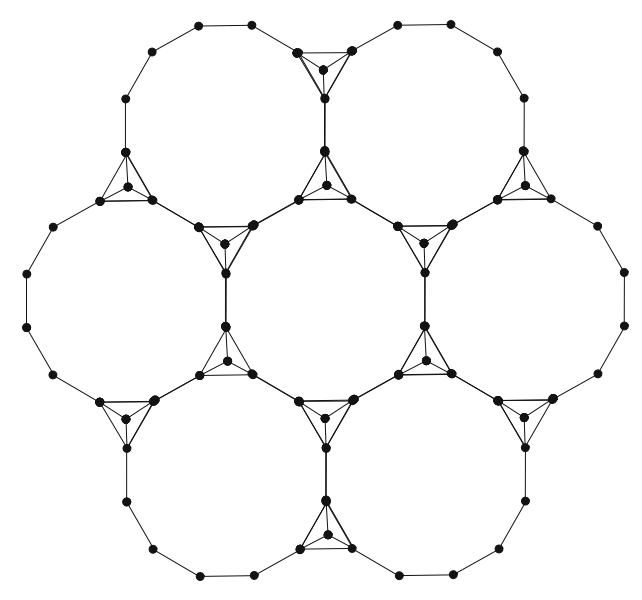

Fig. 11. Periodic graph with the average degree 3.75 .

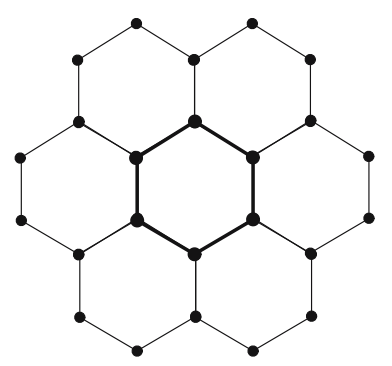

Fig. 12. Periodic graph with the average degree 3.

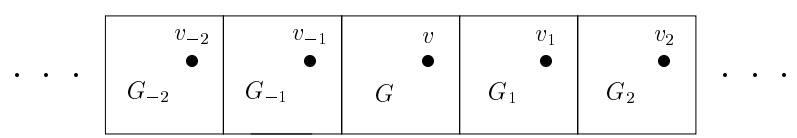

Fig. 13. 1-dimensional, periodic graph generated from $G$.

Proof. Let $G$ be a finite graph, being $M=\mathcal{M}_{G}^{1}$ connected. Let us consider the sequence $\left\{M_{n}(G)\right\}_{n \in \mathbb{N}}$ and $d=\operatorname{diam}(G)$. We want to study the cardinal of $V\left(\partial M_{n}(G)\right)$. To do so, we get $v_{k} \in V\left(G_{k}\right)$ such that $d\left(v_{k}, G\right)_{M}=n$, with $k=\min \left\{i>0: d\left(v_{i}, G\right)=n\right\}$. Consequently, for all $i \geq k+d+1$, we get $d\left(v_{i}, G\right) \geq$ $n+1$, because if we suppose that $d\left(v_{i}, G\right) \leq n$, as $d\left(v_{i}, G_{k}\right) \geq i-k$ (see Fig. 14), there would exist a $w_{k} \in V\left(G_{k}\right)$ such that $d\left(v_{i}, w_{k}\right) \geq i-k$ and, therefore,

$$
\begin{aligned}
d\left(w_{k}, G\right) & \leq d\left(v_{i}, G\right)-d\left(v_{i}, w_{k}\right) \\
& \leq n-i+k \leq n-d-1
\end{aligned}
$$

However,

$$
\begin{aligned}
d\left(v_{k}, G\right) & \leq d\left(v_{k}, w_{k}\right)+d\left(w_{k}, G\right) \\
& \leq d+n-d-1=n-1,
\end{aligned}
$$

and this is not possible. Following analogous reasoning for $k<0$, we get

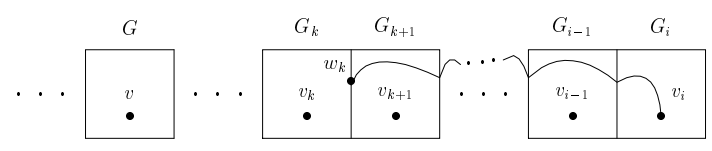

Fig. 14. Path from $v_{i}$ to $G_{k}$ in $M$.

$$
\left|V\left(\partial M_{n}(G)\right)\right| \leq 2(d+1)|V(G)| .
$$

Then

$$
\lim _{n \rightarrow+\infty} \frac{\left|V\left(\partial \mathcal{M}_{n}(G)\right)\right|}{\left|V\left(\mathcal{M}_{n}(G)\right)\right|}=0
$$

because

$$
\lim _{n \rightarrow+\infty}\left|V\left(\mathcal{M}_{n}(G)\right)\right|=+\infty
$$

and the result follows.

Summarizing, we have extended the definition of an average degree, $d_{\infty}(G)$, for a family of infinite graphs which we call average-measurable (see Fig. 15). For instance, trees, periodic graphs, and maximal degree bounded quasi-finite graphs are average-measurable.

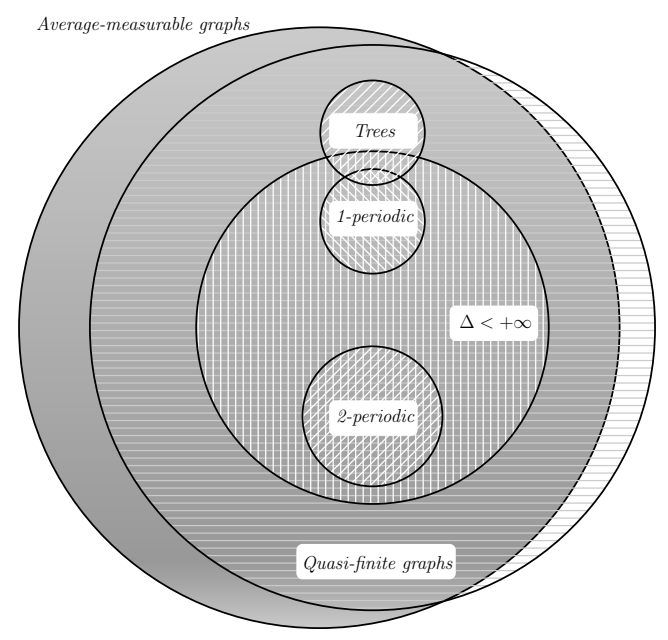

Fig. 15. Relationships among average-measurable graphs.

\section{Extremal problems for infinite graphs}

As stated in Introduction, we are trying to extend some extremal problems to infinite graphs. Actually, we state the study of the function $\operatorname{ex}\left(n ; T K_{p}\right)$ for infinite graphs. Bearing in mind that the study of the number of edges related with the number of vertices does not make any sense for infinite graphs (both are infinite), we could not directly extend the function $\operatorname{ex}\left(n ; T K_{p}\right)$ to infinite graphs. However, the previous function does make sense if we consider each infinite graph as the limit of finite graphs. In other words, given an infinite graph $G$, we are finding any condition related with the function $\operatorname{ex}\left(n ; T K_{p}\right)$, in such 
a way that we may guarantee the existence of a subgraph homeomorphic to $K_{p}$. Since we get an infinite graph as the limit of finite graphs, we need a condition that does not depend on the number of vertices. In this sense, we use the function $d(p)$ to extend the finite case to infinite graphs.

First, we recall that if $H$ is a finite graph with $d(H)>d(p)$, then $H \supseteq T K_{p}$, where

$$
d(p)=\inf \left\{t: \forall H, d(H) \geq t \longrightarrow H \supseteq T K_{p}\right\} .
$$

This concept is easily extendable to infinite graphs.

Definition 6. Let $p$ be a positive integer. We denote by $d_{\infty}(p)$ the function

$$
\begin{gathered}
d_{\infty}(p)=\inf \{t: \forall G \text { average-measurable }, \ldots \\
\left.\ldots d_{\infty}(G) \geq t \longrightarrow G \supset T K_{p}\right\} .
\end{gathered}
$$

In the previous definition, since we used the concept of the average degree, we required that the considered graphs should the average-measurable. But this definition may be extended without restrictions as follows.

Definition 7. Given $p \in \mathbb{N}$, we will denote by $\underline{d}_{\infty}(p)$ and $\bar{d}_{\infty}(p)$ the following two functions:

$$
\begin{aligned}
& \underline{d}_{\infty}(p) \\
= & \inf \left\{t: \forall G \text { infinite, } \underline{d}_{\infty}(G) \geq t \longrightarrow G \supset T K_{p}\right\}
\end{aligned}
$$

and

$$
\begin{aligned}
& \bar{d}_{\infty}(p) \\
= & \inf \left\{t: \forall G \text { infinite, } \bar{d}_{\infty}(G) \geq t \longrightarrow G \supset T K_{p}\right\} .
\end{aligned}
$$

As a conclusion of the previous definitions, it is obvious that $d_{\infty}(p) \leq \underline{d}_{\infty}(p)$, because if $G$ is an infinite average-measurable graph and

$$
t \in\left\{t: \forall G \text { infinite, } \underline{d}_{\infty}(G) \geq t \longrightarrow G \supset T K_{p}\right\},
$$

then if $d_{\infty}(G) \geq t$, as $\underline{d}_{\infty}(G)=d_{\infty}(G) \geq t$, we have that $T K_{p} \subset G$. That is to say,

$$
\begin{aligned}
\left\{t: d_{\infty}(G) \geq t\right. & \left.\longrightarrow G \supset T K_{p}\right\} \\
& \supseteq\left\{t: \underline{d}_{\infty}(G) \geq t \longrightarrow G \supset T K_{p}\right\}
\end{aligned}
$$

and, therefore, by the definition of $d_{\infty}(p)$ and $\underline{d}_{\infty}(p)$, the inequality $d_{\infty}(p) \leq \underline{d}_{\infty}(p)$ is satisfied.

Analogously, since $\underline{d}_{\infty}(G) \leq \bar{d}_{\infty}(G)$, if

$$
t \in\left\{t: \forall G \text { infinite, } \bar{d}_{\infty}(G) \geq t \longrightarrow G \supset T K_{p}\right\},
$$

then if $\underline{d}_{\infty}(G) \geq t$, as $\bar{d}_{\infty}(G) \geq \underline{d}_{\infty}(G) \geq t$, we have that $G \supset T K_{p}$ and, therefore, $\underline{d}_{\infty}(p) \leq \bar{d}_{\infty}(p)$.

Now, we are proving a result where the previous functions are related to the function $d(p)$. This result allows us to affirm that the functions described above are finite and correctly defined.

Theorem 6. Let $p$ be a positive integer. Then

$$
d(p)-1 \leq d_{\infty}(p) \leq \underline{d}_{\infty}(p) \leq \bar{d}_{\infty}(p) \leq d(p) .
$$

Proof. At first, we are going to demonstrate that $d(p)-1 \leq d_{\infty}(p)$. To prove this inequality, it is sufficient to show that, for every finite graph $G$ with $d(G) \geq d_{\infty}(p)+1$, the assertion $G \supset T K_{p}$ is satisfied, and then, by taking into account the definition of $d(p)$, we would have that $d(p) \leq d_{\infty}(p)+1$.

In order to achieve this goal, let $G$ be a finite graph and $u$ and $v$ be two vertices belonging to $G$ and satisfying $d(u, v)=\operatorname{diam}(G)=d$. Let us consider the graph $G^{*}$, defined as

$$
V\left(G^{*}\right)=V(G) \cup\{\alpha, \beta\}
$$

and

$$
E\left(G^{*}\right)=E(G) \cup\{(\alpha, u),(\beta, v)\} .
$$

Finally, let $M=\mathcal{M}_{G}^{1}$ be the 1-periodic graph generated from $G^{*}$ (see Fig. 16).

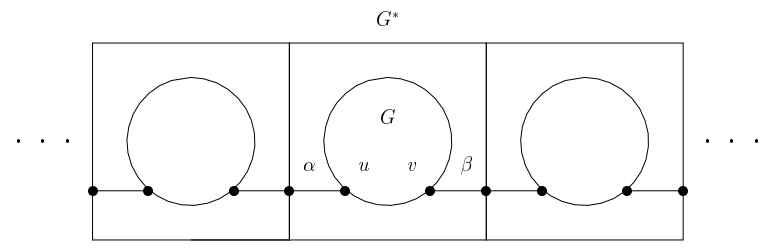

Fig. 16. Graph $M$ generated from $G^{*}$.

By Theorem 5, $M$ is average-measurable, and we therefore consider the subsequence $\left\{M_{(d+2) n}\left(G^{*}\right)\right\}_{n \in \mathbb{N}}$ to calculate its average degree. Since $d_{G^{*}}(\alpha, \beta)=d+2$, we deduce that

$$
\begin{aligned}
\left|V\left(M_{(d+2) n}\left(G^{*}\right)\right)\right| & =\left|V\left(G^{*}\right)\right|+2 n\left(\left|V\left(G^{*}\right)\right|-1\right) \\
& =2+|V(G)|+2 n(|V(G)|+1)
\end{aligned}
$$

and

$$
\begin{aligned}
\left|E\left(M_{(d+2) n}\left(G^{*}\right)\right)\right| & =\left|E\left(G^{*}\right)\right|+2 n\left|E\left(G^{*}\right)\right| \\
& =2+|E(G)|+2 n(|E(G)|+2) .
\end{aligned}
$$

Hence

$$
\begin{aligned}
d_{\infty}(M) & =\lim _{n \rightarrow+\infty} 2 \frac{\left|E\left(M_{(d+2) n}\left(G^{*}\right)\right)\right|}{\left|V\left(M_{(d+2) n}\left(G^{*}\right)\right)\right|} \\
& =2 \lim _{n \rightarrow+\infty} \frac{2+|E(G)|+2 n(|E(G)|+2)}{2+|V(G)|+2 n(|V(G)|+1)} \\
& =2 \cdot \frac{|E(G)|+2}{|V(G)|+1} .
\end{aligned}
$$


Thus,

$$
d_{\infty}(M)=\frac{|E(G)|+2}{|V(G)|+1}>2 \frac{|E(G)|}{|V(G)|}-1 \geq d_{\infty}(p)
$$

because

$$
\begin{aligned}
& \frac{|E(G)|+2}{|V(G)|+1}>2 \frac{|E(G)|}{|V(G)|}-1 \\
& \longleftrightarrow|E(G)|<\frac{|V(G)|^{2}+5|V(G)|}{2} .
\end{aligned}
$$

But

$$
|E(G)| \leq \frac{|V(G)|(|V(G)|-1)}{2}
$$

and

$$
\begin{aligned}
& \frac{|V(G)|(|V(G)|-1)}{2}<\frac{|V(G)|^{2}+5|V(G)|}{2} \\
& \longleftrightarrow|V(G)|>0 .
\end{aligned}
$$

Thus (by the definition of $d_{\infty}(p)$ ), $M \supset T K_{p}$. But, by the construction of $M$, if $M \supset T K_{p}$, then $G \supset T K_{p}$. Thus, if $d(G) \geq d_{\infty}(p)+1$, then $G \supset T K_{p}$, and the inequality $d(p) \leq d_{\infty}(p)+1$ holds.

In order to prove that $\bar{d}_{\infty}(p) \leq d(p)$, we suppose the opposite case. Let $a$ and $b$ be two real numbers such that $\bar{d}_{\infty}(p)>a>b>d(p)$. Let $G$ be an infinite graph and we suppose that $\bar{d}_{\infty}(G) \geq a$. Given $\varepsilon=(a-b) / 2$, by definition of $\bar{d}_{\infty}(G)$, there exists a vertex $v \in G$ such that

$$
\limsup _{n \rightarrow+\infty} d\left(G_{n}(v)\right) \geq \bar{d}_{\infty}(G)-\varepsilon .
$$

Besides, given $\varepsilon$, by the definition of the superior limit, there exits an $n_{0} \in \mathbb{N}$ such that

$$
d\left(G_{n_{0}}(v)\right) \geq \limsup _{n \rightarrow+\infty} d\left(G_{n}(v)\right)-\varepsilon \geq \bar{d}_{\infty}(G)-2 \varepsilon .
$$

Then

$$
d\left(G_{n_{0}}(v)\right) \geq a-a+b>d(p) .
$$

But, by the definition of the function $d(p), G_{n_{0}}(v) \supset$ $T K_{p}$ and, therefore, $G \supset T K_{p}$. Hence, by the definition of $\bar{d}_{\infty}(p)$, we would have $\bar{d}_{\infty}(p) \leq a<\bar{d}_{\infty}(p)$, but this is not possible.

3.1. Exact values for $d_{\infty}(p)$. Returning to the initial aim which had led us to the extension of an extremal graph problem, we pursue exact values for the function $d_{\infty}(p)=$ $d_{\infty}\left(T K_{p}\right)$

Up to now, for the finite case, the exact values of $d(p)$ have only been known for $p \leq 5$, for which $d(p)=$ $2(p-2)$ (see Wagner, 1960; Dirac, 1960; Mader, 1998a). Now, we are going to show that $d_{\infty}(p)=d(p)$ for these values of $p$.

Let $T$ be a tree. According to Theorem 1, $T$ is average-measurable and $d_{\infty}(T)=2$. Besides, $T$ does not contain any subgraph homeomorphic to $K_{3}$, and, therefore, $d_{\infty}(3) \geq 2$. But, by Theorem 6 .

$$
d_{\infty}(3)=\underline{d}_{\infty}(3)=\bar{d}_{\infty}(3)=d(3)=2 .
$$

In order to prove that $d_{\infty}(4)=d(4)$, consider the graph $G$ shown in Fig. 17. $G$ does not contain a subgraph homeomorphic to $K_{4}$. Furthermore, if we denote by $H$ the subgraph generated from $G$ by the vertices $v_{1}, v_{2}, v_{3}, v_{4}$, and consider the sequence $\left\{G_{n}(H)\right\}_{n \in \mathbb{N}}$, we get

$$
\left|V\left(G_{n}(H)\right)\right|=4(n+1)
$$

and

$$
\left|E\left(G_{n}(H)\right)\right|=5+6+8(n-1) .
$$

In consequence,

$$
\lim _{n \rightarrow+\infty} \frac{\left|V\left(\partial G_{n}(H)\right)\right|}{\left|V\left(G_{n}(H)\right)\right|}=\lim _{n \rightarrow+\infty} \frac{4(n+1)-4 n}{4(n+1)}=0 .
$$

Hence, $G$ is average-measurable and

$$
d_{\infty}(G)=\lim _{n \rightarrow+\infty} 2 \cdot \frac{\left|E\left(G_{n}(H)\right)\right|}{\left|V\left(G_{n}(H)\right)\right|}=4
$$

Thus, $d_{\infty}(4) \geq 4$ and, by taking into account that $d(4)=$ 4 and by applying Theorem 6 , we can assert that $d_{\infty}(4)=$ $d(4)=4$.

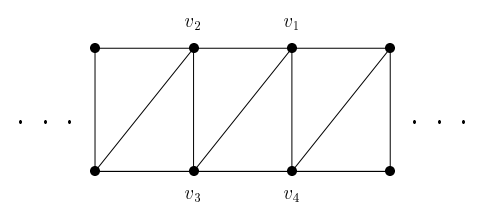

Fig. 17. Average-measurable graph with $d_{\infty}(G)=4$.

For the case $p=5$, we consider the graph $G$ shown in Fig. 18 As this graph admits plane embedding (see Fig. 19], and by applying the Kuratowski theorem for infinite graph 1 (see Dirac and Schuster, 1954): $G \not \supset$ $T K_{5}$.

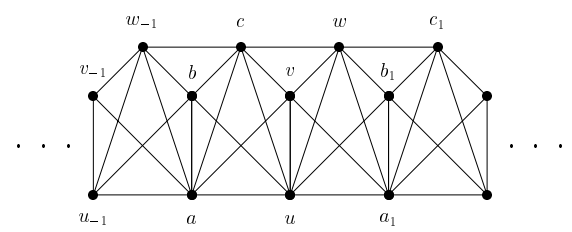

Fig. 18. Average-measurable graph with $d_{\infty}(G)=5$.

We are proving that $G$ is average-measurable and $d_{\infty}(G)=6$. To this end, we consider the induced subgraph $H=\langle\{a, b, c, u, v\}\rangle_{G}$ and the $\operatorname{ICS}\left\{G_{n}(H)\right\}$ :

$$
\left|V\left(G_{n}(H)\right)\right|=5+6 n
$$

\footnotetext{
${ }^{1}$ An infinite, connected graph is plane if and only if all its finite subgraphs are plane.
} 


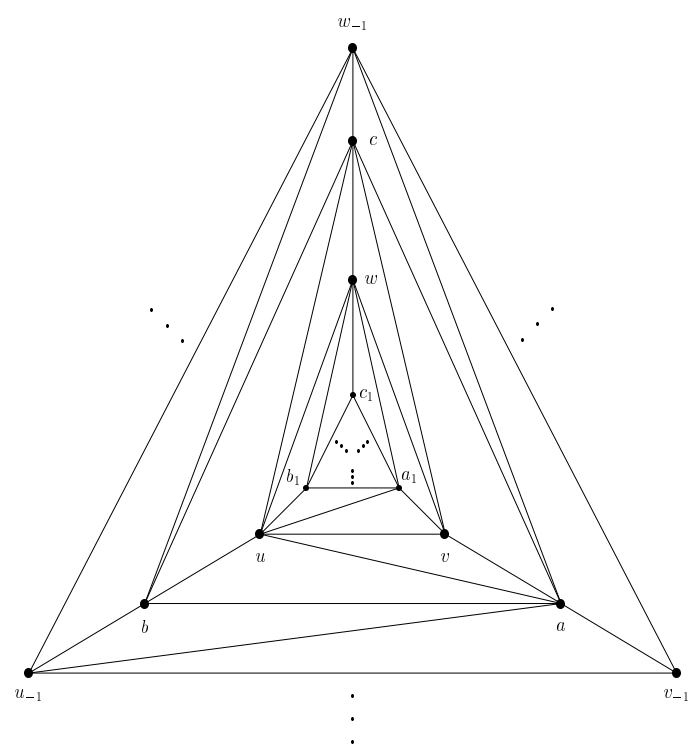

Fig. 19. Planar representation of the graph from Fig. 18

and

$$
\left|E\left(G_{n}(H)\right)\right|=9+18 n
$$

so

$$
\lim _{n \rightarrow+\infty} \frac{\left|V\left(\partial G_{n}(H)\right)\right|}{\left|V\left(G_{n}(H)\right)\right|}=\lim _{n \rightarrow+\infty} \frac{6}{5+6 n}=0
$$

and, therefore, $G$ is average-measurable and

$$
d_{\infty}(G)=\lim _{n \rightarrow+\infty} 2 \cdot \frac{\left|E\left(G_{n}(H)\right)\right|}{\left|V\left(G_{n}(H)\right)\right|}=6 .
$$

Hence, $d_{\infty}(5)=d(5)=6$.

Theorem 7. If $p \leq 5$, then

$$
d_{\infty}(p)=\underline{d}_{\infty}(p)=\bar{d}_{\infty}(p)=d(p)=2(p-2) .
$$

3.2. Generalization of the extremal function. Let $F$ be a finite graph. We define the following functions:

$$
\begin{aligned}
& d(F)=\inf \{t: \forall H \text { finite, } d(H) \geq t \rightarrow H \supseteq F\}, \\
& d_{\infty}(F) \\
= & \inf \left\{t: \forall G \text { average-measurable, } d_{\infty}(G) \geq t \rightarrow G \supset F\right\},
\end{aligned}
$$

$$
\begin{aligned}
& \underline{d}_{\infty}(F) \\
& \quad=\inf \left\{t: \forall G \text { infinite, } \underline{d}_{\infty}(G) \geq t \rightarrow G \supset F\right\}
\end{aligned}
$$

and

$$
\begin{aligned}
& \bar{d}_{\infty}(F) \\
& \quad=\inf \left\{t: \forall G \text { infinite, } \bar{d}_{\infty}(G) \geq t \rightarrow G \supset F\right\} .
\end{aligned}
$$

Then Theorem 6 can be generalized by changing $T K_{p}$ into $F$ in its proof, so the next theorem holds.
Theorem 8. Let $F$ be a finite graph. Then

$$
d(F)-1 \leq d_{\infty}(F) \leq \underline{d}_{\infty}(F) \leq \bar{d}_{\infty}(F) \leq d(F) .
$$

This result allows us to state any forbidden-subgraph problem for infinite graphs as an extension of the problem for the finite case.

\section{Applications to the transportation of dangerous goods}

The origin of this paper dates back to the time when the authors were asked to solve the problem of the location of parking areas for dangerous goods in Europe. As graph theory is particularly related to networks (Diestel, 2000; Péter, 2012; Peng et al., 2013; Kudělka et al., 2015), we tried to solve the proposed questions with the help of graphs.

In our case, when modeling a road transport network by using a graph, vertices (or nodes) usually correspond to crossroads, while edges represent road sections (as in the work of Caro et al. (2015)). In fact, there are several papers where the interest focuses on placing a specific service for drivers inside an edge (see, e.g., Peng et al., 2013; Kudělka et al., 2015). Some others deal with the problem of designing a network to improve the global efficiency (see the works of Milková (2009) or Klaučo et al. (2016) for typical examples).

In such situations, the possibility of minimizing the number of roads (i.e., edges) is relevant. This number of roads can be controlled in graph theory by considering the average degree. And, as road networks tend to expand over time, this characteristic is usually translated into the degrees of vertices in an infinite graph, which are closely related to the function $d_{\infty}(p)$ previously introduced.

Furthermore, the number of edges (and, eventually, new services) sometimes has to be locally limited by considering the number of edges incident in each vertex (i.e., their vertex degree). Thus, this is another natural reason to study average degrees in complex graphs modeling real road transport networks. In fact, the average degree has already been used to deal with economic matters (see, e.g., Fedriani et al., 2005; Ruiz et al., 2008) but it has only been defined for finite graphs. For this reason, its properties have not been used in gradually increasing models, such as those obtained when modelling increasing networks. Two typical cases of graphs that appear when dealing with these networks are periodic graphs (if a structural design has to be replicated), and maximal degree bounded quasi-finite graphs (for reasons mentioned above). Note that we have proved that both the families are average-measurable, and we have provided a way of computing the 'average degree' for these infinite cases. Even more, the other average-measurable family given in this paper (trees) is also crucial when applying topological or combinatorial algorithms to 
network problems; examples include solving problems on finding the shortest path connecting a set of nodes or vertices, depending on minimum spanning trees or Steiner trees.

On the other hand, from a practical point of view, it is also essential to consider some other characteristics which can be related to the presence of certain complete graphs (i.e., graphs including all the possible edges between their vertices) and other structures (e.g., Balaji and Revathi, 2012; Li, 2012; Li et al., 2012) in road transportation models. For example, the presence of $K_{3}$ implies the existence of a cycle which will, in all likelihood, not be necessary to guarantee communication and transport. The presence of a $K_{4}$ might affect the future development of the network since it goes against outerplanarity. The presence of $K_{5}$ prevents the network from being a design without intersections. Most of the problems described above can be modeled from the point of view of extremal graph theory we have considered in this paper, since $d(p)$ and $\operatorname{ex}\left(n ; T K_{p}\right)$ refer to the possibility of having a complete graph $K_{p}$ as a subgraph inside the network. Hence, we maintain that the search of complete graphs in networks can complement other approaches (see, e.g., Dridi and Kacem, 2004; Péter, 2012). However, we think that the greatest benefit of our generalization of the average degree comes from the fact that it is useful to model complex, increasing networks.

In a different context, percolation models are infinite random graph models for phase transitions and critical phenomena. Percolation is also a key concept when dealing with hazardous issues such as those concerning dangerous goods (from the analysis of explosions to epidemic spreading models). The determination of the critical probability (or the percolation threshold) for the percolation model is one of the most interesting problems in this area (see, e.g., Stauffer and Aharony, 1992; Sahimi, 1994), and it depends on the universal formulas introduced by Wierman and Naor (2005). In fact, most of the related formulas cited in the physics and engineering literature are based on the average degree of infinite graphs (see Wierman and Naor, 2005; Wierman et al., 2005). However, as the average degree of infinite graphs was only well defined for infinite 2-dimensional periodic graphs, the exact percolation threshold was only known for a few arbitrary trees and infinite 2-dimensional periodic graphs. The results presented in this paper generalize the notion of an average degree to another family of infinite graphs, allowing the universal formulas to be used for them.

\section{Conclusion}

We have presented a theoretical definition of the average degree $d_{\infty}(G)$ for a family of infinite graphs which is useful in problems related to the transportation of dangerous goods (road transportation networks and the percolation threshold). This concept was introduced by starting with a sequence of finite graphs, in such a way that the average degree for infinite graphs inherits the properties of the average degree for the finite case. Taking these assertions into account, we gave a definition of the average degree for average-measurable, infinite graphs. Trees, periodic graphs, and maximal degree bounded quasi-finite graphs are average-measurable graphs.

On these average-measurable infinite graphs, we extended the function $d(p)$ (in general, $d(F)$ ) to infinite graphs, as $d_{\infty}(p)$, and we linked it to the finite case, showing that $d(p)-1 \leq d_{\infty}(p) \leq d(p)$ (the same for $d(F)$ ). Moreover, we proved that $d_{\infty}(p)=d(p)=$ $2(p-2)$ for $p \leq 5$.

Finally, we noted the applicability of theoretical concepts on infinite graphs to the transportation of dangerous goods.

\section{Acknowledgment}

The authors would like to thank the European Union ERDF for financial support via the project Localización de áreas de estacionamiento para mercancías peligrosas en Andalucía y la Red Transeuropea of Programa Operativo FEDER de Andalucía 2007-2013. We also thank the Public Works Agency and the Regional Ministry of Public Works and Housing of the Regional Government of Andalusia, and the two anonymous referees for their helpful suggestions.

\section{References}

Balaji, S. and Revathi, N. (2012). An efficient approach for the optimization version of maximum weighted clique problem, WEAS Transactions on Mathematics 11(9): 773-783.

Barooah, P. and Hespanha, J. (2008). Estimation from relative measurements: Electrical analogy and large graphs, IEEE Transactions on Signal Processing 56(6): 2181-2193.

Bauderon, M. (1989). On system of equations defining infinite graphs, in J. van Leeuwen (Ed.), Graph-Theoretic Concepts in Computer Science, Lecture Notes in Computer Science, Vol. 344, Springer-Verlag, Berlin/Heidelberg, pp. 54-73.

Caro, M., Fedriani, E. and Tenorio, A. (2015). Design of an efficient algorithm to determine a near-optimal location of parking areas for dangerous goods in the European Road Transport Network, in F. Corman et al. (Eds.), ICCL 2015, Lecture Notes in Computer Science, Vol. 9335, Springer International Publishing, Cham, pp. 617-626.

Cayley, A. (1895). The theory of groups, graphical representation, Cambridge Mathematical Papers 10: $26-28$.

Cera, M., Diánez, A. and Márquez, A. (2000). The size of a graph without topological complete subgraphs, SIAM Journal on Discrete Mathematics 13(3): 295-301. 
Cera, M., Diánez, A. and Márquez, A. (2004). Extremal graphs without topological complete subgraphs, SIAM Journal on Discrete Mathematics 18(2): 288-396.

Diestel, R. (2000). Graph Theory, Springer-Verlag, Berlin/Heidelberg.

Dirac, G. (1960). In abstrakten Graphen vorhandene vollständige 4-Graphen und ihre Unterteilungen, Mathematische Nachrichten 22: 61-85.

Dirac, G. and Schuster, S. (1954). A theorem of Kuratowski, Indagationes Mathematicae 16: 343-348.

Dridi, M. and Kacem, I. (2004). A hybrid approach for scheduling transportation networks, International Journal of Applied Mathematics and Computer Science 14(3): 397-409.

Fedriani, E., Mínguez, N. and Martín, A. (2005). Estabilidad de los indicadores topológicos de pobreza, Rect@ 13(1), Record No. 39.

Frucht, R. (1938). Herstellung von Graphen mit vorgegebener abstrakten Gruppe, Compositio Mathematica 6: 239-250.

Grünbaum, B. and Shephard, G. (1987). Tiling and Patterns, Freeman, New York, NY.

Klaučo, M., Blažek, S. and Kvasnica, M. (2016). An optimal path planning problem for heterogeneous multi-vehicle systems, International Journal of Applied Mathematics and Computer Science 26(2): 297-308, DOI: 10.1515/amcs-2016-0021.

Kudělka, M., Zehnalová, S., Horák, Z., Krömer, P. and Snášel, V. (2015). Local dependency in networks, International Journal of Applied Mathematics and Computer Science 25(2): 281-293, DOI: 10.1515/amcs-2015-0022.

Li, F. (2012). Some results on tenacity of graphs, WEAS Transactions on Mathematics 11(9): 760-772.

Li, F., Ye, Q. and Sheng, B. (2012). Computing rupture degrees of some graphs, WEAS Transactions on Mathematics 11(1): 23-33.

Mader, W. (1967). Homomorphieegenshaften und mittlere Kantendichte von Graphen, Mathematische Annalen 174: 265-268.

Mader, W. (1998a). $3 n-5$ edges do force a subdivision of $K_{5}$, Combinatorica 18(4): 569-595.

Mader, W. (1998b). Topological minors in graphs of minimum degree n, DIMACS Series in Discrete Mathematics and Theoretical Computer Science 49: 199-211.

Milková, E. (2009). Constructing knowledge in graph theory and combinatorial optimization, WSEAS Transactions on Mathematics 8(8): 424-434.

Peng, W., Dong, G., Yang, K. and Su, J. (2013). A random road network model and its effect on topological characteristics of mobile delay-tolerant networks, IEEE Transactions Mobile Computing 13(12): 2706-2718.

Péter, T. (2012). Modeling nonlinear road traffic networks for junction control, International Journal of Applied Mathematics and Computer Science 22(3): 723-732, DOI: 10.2478/v10006-012-0054-1.
Ruiz, E., Hernández, M. and Fedriani, E. (2008). The development of mining heritage tourism: A systemic approach, in A.D. Ramos and P.S. Jiménez (Eds.), Tourism Development: Economics, Management and Strategy, Nova Science Publishers, Inc., Hauppauge, NY, pp. 121-143.

Sahimi, M. (1994). Applications of Percolation Theory, Taylor and Francis, London.

Stauffer, D. and Aharony, A. (1992). Introduction to Percolation Theory, Taylor and Francis, London.

Stein, M. (2011). Extremal infinite graph theory, Discrete Mathematics 311(15): 1472-1496.

Stein, M. and Zamora, J. (2013). Forcing large complete (topological) minors in infinite graphs, SIAM Journal on Discrete Mathematics 27(2): 697-707.

Wagner, K. (1960). Bemerkungen zu Hadwigers Vermutung, Mathematische Annalen 141: 433-451.

Wierman, J. and Naor, D. (2005). Criteria for evaluation of universal formulas for percolation thresholds, Physical Review E 71(036143).

Wierman, J., Naor, D. and Cheng, R. (2005). Improved site percolation threshold universal formula for two-dimensional matching lattices, Physical Review E 72(066116).

Yang, Y., Lin, J. and Dai, Y. (2002). Largest planar graphs and largest maximal planar graphs of diameter two, Journal of Computational and Applied Mathematics 144(1-2): 349-358.

Yousefi-Azaria, H., Khalifeha, M. and Ashrafi, A. (2011). Calculating the edge Wiener and edge Szeged indices of graphs, Journal of Computational and Applied Mathematics 235(16): 4866-4870.

Zemanian, A. (1988). Infinite electrical networks: A reprise, IEEE Transactions on Circuits and Systems 35(11): 1346-1358.

Martín Cera works as a lecturer in the Department of Applied Mathematics I at the University of Seville, Spain. He is a researcher at the university's Antonio de Castro Brzezicki Institute of Mathematics (IMUS). His area of interest includes extremal graph theory, social network analysis, mathematical biology and transportation networks analysis. He has authored/co-authored several refereed journal/conference papers, books and book chapters

Eugenio M. Fedriani is a lecturer (with full professor certification) in the Department of Economics, Quantitative Methods, and Economic History at Pablo de Olavide University. He is currently the vice president for academic planning in his university. He obtained his Ph.D. in mathematics in 2001, and his main research interests are topological graph theory, poverty measurement, lie theory, and mathematical education. He has published more than 100 scientific papers and books.

Received: 29 September 2015 Revised: 17 April 2016

Re-revised: 3 June 2016 Accepted: 2 July 2016 